\title{
Loss of controllability in appetitive situations interferes with subsequent learning in aversive situations
}

\author{
AKIHITO SONODA, TAKAHIRO OKAYASU, and HISASHI HIRAI \\ Sophia University, Tokyo, Japan
}

\begin{abstract}
In two experiments, we examined whether or not a loss of control over food availability would interfere with subsequent two-way shuttle-escape learning. Rats that had experienced loss of control over food delivery were impaired in their acquisition of a shuttle-escape response, relative to the response-contingent and the continuous-reinforcement control rats (in Experiments 1 and 2) and to the lack-of-control and home cage control rats (in Experiment 2). Rats that had received noncontingent food delivery without a prior history of control over food exhibited poorer performance than did the home cage control rats. Moreover, loss of control resulted in a larger interference effect than did lack of control, supporting the view that the learning of response-outcome noncontingency is the main determinant of the interference effect.
\end{abstract}

Since Overmier and Seligman (1967; Seligman \& Maier, 1967) found that exposure to inescapable shock interferes with later learning, many studies of this effect have been conducted. It has been termed the learned helplessness effect (Seligman, 1975) or the interference effect (Glazer \& Weiss, 1976a, 1976b). The learned helplessness theory (Maier \& Seligman, 1976; Seligman, 1975) explains that this effect arises from the learning of uncontrollability-that outcomes are independent of responses. This type of learning may cause motivational, associative, and emotional deficits. The learned helplessness theory has also been termed a "cognitive theory" by its proponents (Maier \& Seligman, 1976).

Three prominent, alternative hypotheses about this phenomenon exist. These are the competing motor response hypothesis (Levis, 1976; Glazer \& Weiss, 1976a, 1976b), the fear hypothesis (Jackson \& Minor, 1988; Minor \& LoLordo, 1984), and the norepinephrine-depletion hypothesis (Anisman, 1975; Weiss et al., 1981). The competing motor response hypothesis states that during an inescapable shock, inactivity is associated with and reinforced by the shock offset, and that activity is consequently reduced during the test task, which requires active responses to escape from shock. The fear hypothesis states that a high level of fear induced by inescapable shock might be responsible for behavioral deficits. The norepinephrinedepletion hypothesis states that the stress of uncontrollable

We wish to express our thanks to Akira Tsuda of the Kurume University School of Medicine for his invaluable advice and encouragement. We also wish to express our thanks to Gary B. Glavin of the University of Manitoba for his kind reviewing of an earlier version of this manuscript. Takahiro Okayasu is now at Waseda University in Saitama, Japan. Correspondence concerning this article should be addressed to Akihito Sonoda, Department of Psychology, Sophia University, Tokyo 102, Japan. shock causes a depletion of norepinephrine, which results in an inability to produce motor responses required for the task at hand.

Until now, many researchers have found evidence in support of the learned helplessness theories by using appetitive test tasks (Plonsky, Warren, \& Rosellini, 1984; Rosellini, 1978; Rosellini \& DeCola, 1981; Rosellini, DeCola, \& Shapiro, 1982). For example, Rosellini et al. (1982) found that exposure to inescapable shock interferes with subsequent nose-poke discrimination acquisition in appetitive situations, but not with the activity itself. This suggests that associative factors, but not activity deficits, cause the interference effects. Sonoda (1990) also found the interfering effects of exposure to inescapable shocks on an appetitive barpress discrimination task. However, some experiments have yielded a possible alternative to appetitive helplessness (e.g., Dess, Chapman, \& Minor, 1988).

The most prominent experimental paradigm supporting the associative theory involves response-independent food presentation in the first phase. Seligman (1975) and Maier and Seligman (1976) have claimed that uncontrollability in appetitive situations may also cause interference effects. At present, interference effects arising from response-independent food have been reported in many studies (e.g., Calef et al., 1989; Calef, Murray, Modlin, Meekins, \& Geller, 1981; Goodkin, 1976; Job, 1987, 1988, 1989; Oakes, Rosenblum, \& Fox, 1982), but some investigators have failed to report such an effect (Beatty \& Maki, 1979; Calef et al., 1984). It is noteworthy that some of this research (e.g., Job, 1987; Oakes et al., 1982) has not yielded motivational deficits, but rather associative ones, in discrimination tests. These results suggest that the interference effect is due to an associative factor.

The present study represents an attempt to determine whether the loss of controllability in appetitive situations 
results in interference effects in aversive situations. Maier, Seligman, and Solomon (1969) gave a probabilistic definition of uncontrollability, by saying that the probability of an outcome, given a response, must be equal to the probability of an outcome, given no response. In many experiments on appetitive uncontrollability, rats have been presented with response-noncontingent food from the beginning of the experiment. In such cases, the yoked rats do not perform a target response, such as a leverpress, from the beginning of the experiment. Therefore, these rats have not experienced their target response as adventitiously producing food delivery and then producing no food. The loss of controllability over an appetitive outcome, given a prior history of control, maintains the leverpress response during response-independent food pretreatment. Therefore, these rats experience their leverpress response as adventitiously producing food delivery and then producing no food, and they learn noncontingency between a target response and a food outcome.

Although some experiments with appetitive uncontrollability have methodological defects, such as, for example, the use of the same response in both the pretreatment and the test phases (e.g., Caspy \& Lubow, 1981) and the exclusion of an untreated control group (e.g., Wight \& Katzev, 1977), in the present experiment we avoided these defects through the use of a yoked design, as well as the use of different responses in the pretreatment and test phases.

\section{EXPERIMENT 1}

Our main interest in Experiment 1 was whether the loss of controllability in appetitive situations, represented by the learning of noncontingency between a target response and a food outcome, produces an interference effect in an aversive situation.

Method
Subjects
Twenty-seven male naive Wistar rats, 9 weeks old (each weigh-
ing approximately $280 \mathrm{~g}$ at the beginning of the study), were used
as the subjects. All the animals were housed in group cages before
the experimental sessions. Once the experimental sessions began,
they were housed individually in other cages. They were maintained
on a 12:12-h light:dark cycle with water ad lib.

\section{Apparatus}

Three identical Skinner boxes and one shuttlebox were used. The Skinner boxes employed in continuous reinforcement (CRF) training and pretreatment consisted of a chamber with clear Plexiglas sidewalls and ceiling, stainless steel front and rear walls, and a floor of stainless steel rods $(1.6 \mathrm{~cm}$ center to center). Internally, boxes were $23.5 \mathrm{~cm}$ wide, $25.5 \mathrm{~cm}$ high, and $30.0 \mathrm{~cm}$ long. The front walls had an attached food cup, $1.0 \mathrm{~cm}$ in diameter, located $2.5 \mathrm{~cm}$ above the floor and $12.5 \mathrm{~cm}$ from the right sidewall. A $2-\mathrm{cm}$ long, $3-\mathrm{cm}$ wide stainless steel bar was located $4.0 \mathrm{~cm}$ from the right sidewall, $5.5 \mathrm{~cm}$ above the floor. Barpresses were automatically detected and recorded by a personal computer PC-8801 or PC-9801, and $45-\mathrm{mg}$ food pellets (Holton Industries) were delivered by a pellet dispenser.
The escape test was administered in a $46 \times 20 \times 20 \mathrm{~cm}$ twoway shuttlebox consisting of clear Plexiglas sidewalls, stainless steel end walls, and a grid floor (with rods $1.1 \mathrm{~cm}$ center to center). The center of the shuttlebox contained a metal divider with a rounded archway $9.5 \mathrm{~cm}$ high and $6.3 \mathrm{~cm}$ wide cut out of it. Scrambled shocks ( $1.5 \mathrm{~mA}$, peak voltage of $40 \mathrm{~V} \mathrm{dc}$ ) were delivered to the grid floors with an SGS-001 shock generator/scrambler (Muromachi Kikai Co.). The experiment was controlled by a personal computer PC8801 or PC-9801

\section{Procedure}

The animals were placed on a 23-h food-deprivation schedule, with access to food during $1 \mathrm{~h}$ per day, over a 5-day period prior to the experimental session. This reduced their weight by approximately $15 \%$ prior to the beginning of the experiment. The experiment was conducted in nine replications of triplets.

CRF training. On the 1st day of the experiment, in order to make the rats acquire controllability, all were administered one CRF training session in which they earned 100 pellets by barpressing in the Skinner box. The elapsed time to earn the pellets during this session was used for the assignment of the animals to each of the three pretreatment conditions, in such a way that elapsed time was equal among the groups.

Pretreatment. The rats were assigned to one of three pretreatment groups ( $n=9$ per group) in a typical learned helplessness triadic design that included the response-contingent group, the lossof-control group, and the CRF control group. The responsecontingent group could earn a food pellet by barpressing on a fixedratio (FR) 5 schedule on the 2 nd day of the experiment, and on an FR20 on the 3rd day. The rats were allowed to earn 100 pellets per day. The loss-of-controllability group was yoked to the responsecontingent group and thus received pellets at the same time as the response-contingent rats did, except that the pe!let delivery was independent of the yoked-noncontingent animals' behavior. The CRF control group was not administered a pretreatment, but 100 pellets, the same number as would be earned by the response-contingent group, were presented en masse on a plate in their home cage at the same time as the other groups had the pretreatment session.

Test. On the 4th day of the experiment, all rats were tested with the two-crossing (FR2) shuttle-escape task. A 1.5-mA shock was delivered until rats crossed the shuttlebox twice. If the rats failed to escape within $60 \mathrm{sec}$ after the shock onset, the trial automatically terminated and a 60 -sec latency was recorded. The intertrial interval had a mean of $40 \mathrm{sec}$, with a range between 20 and $60 \mathrm{sec}$. Fifty trials of the escape task were administered.

\section{Results and Discussion}

\section{CRF Training}

The mean elapsed times to completion of CRF training and the standard deviations for the response-contingent, the loss-of-controllability, and the CRF control groups were $135.7(S D=138.3), 179.6(S D=109.5)$, and $103.3(S D=112.2) \mathrm{min}$, respectively. An analysis of variance (ANOVA) revealed no differences between the groups.

\section{CRF and Pretreatment}

The mean number of responses during the last $5 \mathrm{~min}$ is shown in Figure 1 for each treatment (CRF, FR5, and FR20). The number of responses during the last $5 \mathrm{~min}$ may reflect the effects of each treatment. An ANOVA revealed reliable effects of groups $[F(1,16)=73.72$, $p<.01]$ and treatment $[F(2,32)=20.84, p<.01]$, and 


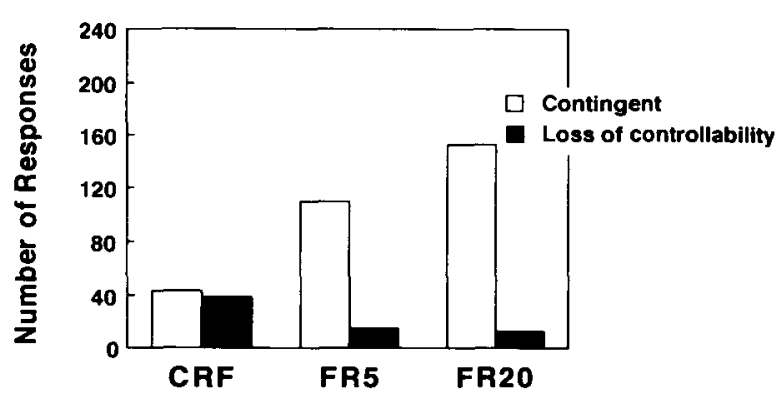

Figure 1. Mean number of responses during the last $5 \mathrm{~min}$ on the CRF, FR5, and FR20 sessions.

a reliable interaction of these factors $[F(2,32)=58.22$, $p<.01]$. The simple main effect of groups on the CRF treatment was not significant, whereas all other simple main effects, including those of groups on the FR5 and FR20 treatments, and treatments on the contingent and the loss-of-control groups, were significant $(p<.05)$. The reliable effect of treatment on the loss-ofcontrollability group denotes that these rats lost their controllability over food.

Test

The mean response latencies on the shuttle escape task are shown in Figure 2 for each group across blocks of 10 trials. The loss-of-controllability rats failed to acquire the escape response. A groups (3) $\times$ blocks (5) two-way ANOVA, with one factor repeated, revealed reliability in the main effects of group $[F(2,24)=22.31, p<.01]$ and blocks of 10 trials $[F(4,96)=7.44, p<.01]$, as well as in the interaction of groups and blocks $[F(8,96)=7.29$, $p<.01]$. The simple effects of block on each of three groups were significant $(p<.01)$. Subsequent Newman-Keuls comparisons $(p<.01)$ showed that the lossof-controllability group differed from the other two groups, which did not differ from each other. Interestingly, subsequent Newman-Keuls comparisons also revealed that on the second block the response-contingent rats were reliably faster than the control rats.

These results show that the loss of control over food acquisition produces a cross-motivational interference ef-

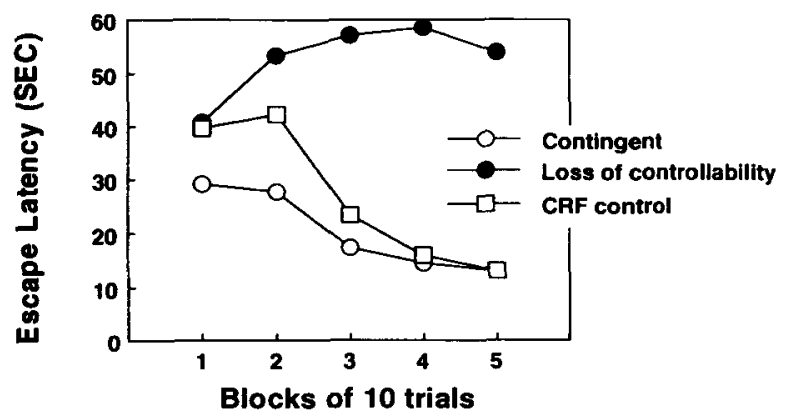

Figure 2. Mean shuttlebox escape latencies across blocks of $\mathbf{1 0}$ trials. fect, comparable to the effects of exposure to uncontrollability in an aversive situation. We suggest that this effect is due to the learning of response-outcome noncontingency through loss of control. However, a direct comparison between the effect of noncontingency through loss of control and the effect of noncontingency through lack of control, given no prior history of control, was not performed. Furthermore, it remained ambiguous whether the difference between the contingent group and the control group on the second block was due to mastery in the contingent group or to retardation in the control group, which might have experienced some loss of control because it received noncontingent food pellets in the home cage after a CRF session. Experiment 2 was designed to address these issues.

\section{EXPERIMENT 2}

The present experiment was conducted to examine the problems noted above by adding the following two groups: a lack-of-controllability group, which received yokednoncontingent food in the experimental room given no prior contingent FR1 training, and a home cage control group, which received a pile of pellets in the home cage on the FR1 day as well as on the FR5 and FR20 days.

\section{Method}

Subjects

The subjects were 40 rats of the same age, sex, and strain as in Experiment 1. Housing conditions were also identical to those of the preceding experiment, except that the rats were housed in group cages throughout the experimental sessions.

\section{Apparatus}

The apparatus was the same as that in Experiment 1, except that the shock intensity during the shuttle escape test was $1.2 \mathrm{~mA}$.

\section{Procedure}

The deprivation schedule was identical to that in Experiment 1.

Shaping and CRF training. The rats were randomly assigned to one of five groups. The response-contingent, the loss-ofcontrollability, and the CRF control groups were shaped to the leverpress response in a Skinner box with $\mathbf{5 0}$ pellets. On the next day, these rats were administered one CRF training session according to the same procedure as that used in Experiment 1. The lack-ofcontrollability and the home cage control groups consumed 50 pellets on the 1st day and 100 pellets on the 2 nd day, which were presented en masse on a plate in the home cages.

Pretreatment. On the next 2 days, the response-contingent group was administered FR5 and FR20 schedules as in Experiment 1. The loss-of-controllability group and the lack-of-controllability group were yoked to the response-contingent group. The CRF control group and the home cage control group were presented with 100 pellets en masse on a plate in the home cages.

Test. On the 5th day of the experiment, all of the rats were tested in the shuttle escape task. The testing procedure was identical to that used in Experiment 1.

\section{Results}

\section{CRF Training}

The mean elapsed time to completion of CRF training and the standard deviation for the response-contingent, 


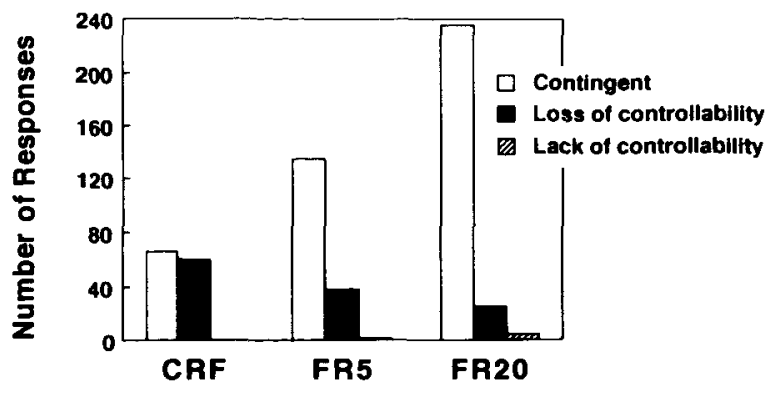

Figure 3. Mean number of responses during the last $5 \mathrm{~min}$ on the CRF, FR5, and FR20 sessions.

the loss-of-controllability, and the CRF control groups were $532.0(S D=112.7), 602.3(S D=179.5)$, and $606.5(S D=205.8) \mathrm{sec}$, respectively. An ANOVA revealed no differences between the groups.

\section{Pretreatment}

The mean number of responses during the last $5 \mathrm{~min}$ is shown in Figure 3 for each treatment (CRF, FR5, and FR20). ANOVAs of group on the FR5 session and on the FR 20 session were significant $[F(2,21)=90.79$, $p<.01, F(2,21)=176.35, p<.01$, respectively] ANOVAs of treatment on the response-contingent and the loss-of-controllability groups also were significant $[F(2,14)=99.92, p<.01, F(2,14)=17.28, p<.01$, respectively].

\section{Test}

The mean response latencies on the shuttle-escape task are shown in Figure 4 for each group across blocks of 10 trials. A groups (5) $\times$ blocks (5) two-way ANOVA, with one factor repeated, revealed reliability in the main effects of groups $[F(4,35)=22.24, p<.01]$ and blocks of 10 trials $[F(4,140)=5.37, p<.01]$, and in the interaction between groups and blocks $[F(16,140)=2.90$, $p<.01]$. Subsequent Newman-Keuls comparisons $(p<.05)$ showed that the loss-of-controllability group differed from the other four groups, and that the lack-ofcontrollability group differed from the home cage control group.

\section{GENERAL DISCUSSION}

The present study was undertaken to explore the effects of loss of controllability in appetitive situations on the performance of rats in aversive situations. The results show that the loss of control over food produces the learning of response-outcome noncontingency and causes subsequent interference effects, to an extent similar to that of the effects of exposure to uncontrollability in an aversive situation. Thus, these results suggest that the learning of response-outcome noncontingency is a major determinant of the interference effect.

The rats with a lack of controllability showed only minor interference effects in Experiment 2. However, it seems that if such rats experience many sessions of noncontingent food, deleterious effects will occur. As evidence of this, other studies with lack of controllability have many days of noncontingent pretreatment-for example, 8 days with 980 pellets in the Job studies (1987, 1988,1989 ), and 9 days in the Oakes et al. report (1982), while in the present experiment there were only 2 days with a total of 200 pellets for the noncontingent phase. This result also indicates that the loss of controllability over food acquisition results in easier learning of a response-outcome noncontingency and is more effective in producing an interference effect than is the lack of controllability over food.

The difference between the contingent group and the CRF control group in Experiment 1 did not appear in Experiment 2; both groups exhibited the same performance as was seen in the home cage control group. If one compares both results, the difference in Experiment 1 seems to be of the nature of a slight performance retardation in the CRF control rats, because the performance of the contingent rats in Experiment 1 was the same as that seen in the two control groups in Experiment 2. One possible cause of this performance retardation might be the stress-

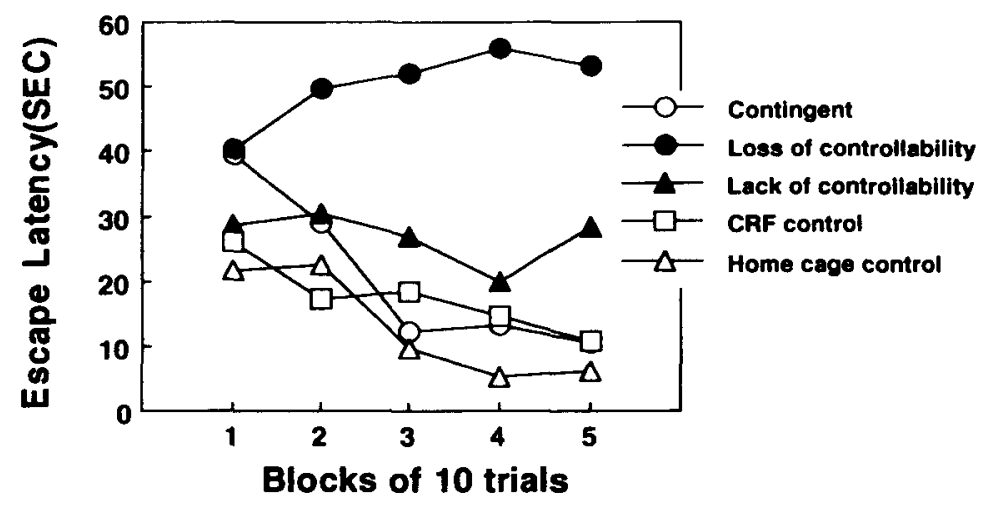

Figure 4. Mean shuttlebox escape latencies across blocks of 10 trials. 
ful influence of the individual housing conditions throughout the experimental session. However, further investigation of this possibility is needed.

One aim in the present experiment was to examine whether the learning of noncontingency between a target response (leverpress) and a food outcome would result in interference effects. The loss-of-controllability procedure has the benefit of making very clear the noncontingency between a target response and a food outcomethe target responses are maintained for the loss-ofcontrollability rats during the pretreatment session. If there is no experience of previous controllability, the noncontingent subject will make few, if any, target responses. In fact, the lack-of-controllability rats made few leverpress responses during the pretreatment phase. A leverpress response by the loss-of-controllability rats was thus sometimes followed by food, adventitiously. The important difference between the contingent rats and the lossof-controllability rats is whether or not a food outcome occurred when no target response was given. Food never occurred for the response-contingent rats when no target response was given, whereas food occurred when the lossof-controllability rats gave no target response as well as when a target response was given. Therefore, the lossof-controllability rats lost the contingency between a target response and a food outcome. Hence, the interference effects in the present two experiments suggest that the cognition of the contingency between a response and an outcome is an important factor in governing an organism's behavior.

Some investigators have reported that prior controllability produces immunization effects (Mullins \& Winefield, 1977; Seligman, Rosellini, \& Kozak, 1975; Warren, Rosellini, Plonsky, \& DeCola, 1985; Williams \& Maier, 1977). In studies showing the immunization effect with aversive situations, initial coping responses are never allowed to occur during inescapable shock. In contrast, in the present study, loss of controllability discouraged the previous coping response during the noncontingent phase. Therefore, the organism lost controllability when such a coping conflict occurred. In fact, much evidence seems to support this interpretation (Hanson, Larson, \& Snowdon, 1976; Tsuda, Ida, \& Tanaka, 1989; Tsuda, Tanaka, Nishikawa, \& Hirai, 1983; Weiss, 1971).

Cross-motivational transfer from an appetitive to an aversive situation was confirmed in the present two experiments. Goodkin (1976) also found such an effect, using response-independent food prior to nose-press escape/ avoidance. In spite of the fact that the reinforcing event was changed from food to shock, the interference effects were observed in this and in the present yoked-noncontingent rats. This fact again suggests that the interference effects are due to the learning of response-outcome independence, and it therefore supports the learned helplessness hypothesis (Maier \& Seligman, 1976; Seligman, 1975).
The present study also shows that interference occurred when a different apparatus was used during the pretreatment and test situations. The pretreatment mainpulandum was absent during testing, and the testing location was absent during the pretreatment. Job (1988) showed that the interference effect in the appetitive situation readily occurs when subjects are preexposed to the manipulandum to be used in the test stage and when the manipulandum employed during pretreatment is absent from the test stage. The present study suggests that it is not a necessary condition of the interference effect that the test manipulandum be present during the pretreatment.

\section{REFERENCES}

ANISMAN, H. (1975). Time-dependent variations in aversively motivated behaviors: Nonassociative effects of cholinergic and catecholaminergic activity. Psychological Review, 82, 359.385.

Beatty, W. W., \& Makı, W. S. (1979). Acquisition of instrumental responding following noncontingent reinforcement: Failure to observe "learned laziness" in rats. Bulletin of the Psychonomic Society, 13, 268-271.

Calef, R. S., Choban, M. C., Dickson, M. W., Newman, P. D., Boyle, M., BAXA, N. D., \& Geller, E. S. (1989). The effects of noncontingent reinforcement on the behavior of a previously learned running response. Bulletin of the Psychonomic Society, 27, 263-266.

Calef, R. S., Metz, R. A., Atkinson, T. L., Pellerzi, R. C., TayLOR, K. S., Geller, E. S. (1984). Acquisition of running in the straight alley following experience with response-independent food. Bulletin of the Psychonomic Society, 22, 67-69.

Calef, R. S., Murray, D. W., Modlin, P. D., Meekins, B. W., a Gler, E. S. (1981). The effects of controllability on extinction. Bulletin of the Psychonomic Society, 17, 241-243.

CASPY, T., \& LUBow, R. E. (1981). Generality of US preexposure effects: Transfer from food to shock or shock to food with and without the same response requirements. Animal Learning \& Behavior, 9 , 524-532.

Dess, N. K., Chapman, C. D., \& Minor, T. R. (1988). Inescapable shock increases finickiness about drinking quinine-adulterated water in rats. Learning \& Motivation, 19, 408-424.

Glazer, H. I., WEISS, J. M. (1976a). Long-term and transitory interference effect. Journal of Experimental Psychology: Animal Behavior Processes, 2, 191-201.

GlAzER, H. I., \& WEISS, J. M. (1976b). Long-term interference effect: An alternative to "learned helplessness." Journal of Experimental Psychology: Animal Behavior Processes, 2, 202-213.

GoodkIN, F. (1976). Rats learn the relationship between responding and environmental events: An expansion of the learned helplessness hypothesis. Learning \& Motivation, 7, 382-393.

Hanson, J. D., Larson, M. E., \& Snowdon, C. T. (1976). The effects of control over high intensity noise on plasma cortisol levels in rhesus monkeys. Behavioral Biology, 16, 333-340.

JACKSON, R. L., \& MINOR, T. R. (1988). Effects of signaling inescapable shock on subsequent escape learning: Implications for theories of coping and "learned helplessness." Journal of Experimental Psychology: Animal Behavior Processes, 14, 390-400.

JoB, R. F. S. (1987). Learned helplessness in an appetitive discretetrial T-maze discrimination test. Animal Learning \& Behavior, 15, 342-346.

JoB, R. F. S. (1988). Interference and facilitation produced by noncontingent reinforcement in the appetitive situation. Animal Learning \& Behavior, 16, 451-460.

JoB, R. F. S. (1989). A test of proposed mechanisms underlying the interference effect produced by noncontingent food presentation. Learning \& Motivation, 20, 153-177

LEVIS, D. J. (1976). Learned helplessness: A reply and an alternative 
S-R interpretation. Journal of Experimental Psychology: General, 105, 47-65.

Maier, S. F., \& Seligman, M. E. P. (1976). Learned helplessness: Theory and evidence. Journal of Experimental Psychology: General, 105, 3-46.

Maier, S. F., Seligman, M. E. P., \& Solomon, R. L. (1969). Pavlovian fear conditioning and learned helplessness: Effects on escape and avoidance behavior of (a) the CS-US contingency and (b) the independence of the US and voluntary responding. In B. A. Campbell \& R. M. Church (Eds.), Punishment and aversive behavior (pp. 299342). New York: Appleton-Century-Crofts.

Minor, T. R., \& LoLORDO, V. M. (1984). Escape deficits following inescapable shock: The role of contextual odor. Journal of Experimental Psychology: Animal Behavior Processes, 10, 168-181.

Mullins, G. P., \& Winefie LD, A. H. (1977). Immunization and helplessness phenomena in the rat in a nonaversive situation. Animal Learning \& Behavior, 5, 281-284.

OAKes, W. F., Rosenblum, J. L., \& Fox, P. E. (1982). "Manna from heaven": The effect of noncontingent appetitive reinforcers on learning in rats. Bulletin of the Psychonomic Society, 19, 123-126.

Overmier, J. B., \& Seligman, M. E. P. (1967). Effects of inescapable shock upon subsequent escape and avoidance responding. Journal of Comparative \& Physiological Psychology, 63, 28-33.

Plonsky, M., Warren, D. A., Rosellini, R. A. (1984). The effects of inescapable shock on appetitive motivation. Bulletin of the Psychonomic Society, 22, 229-231.

RoSELLINI, R. A. (1978). Inescapable shock interferes with the acquisition of an appetitive operant. Animal Learning \& Behavior, 6, 155-159.

Rosellini, R. A., \& DeCola, J. P. (1981). Inescapable shock interferes with the acquisition of a low-activity response in an appetitive context. Animal Learning \& Behavior, 9, 487-490.

Roseleini, R. A., DeCola, J. P., \& Shapiro, N. R. (1982). Crossmotivational effects of inescapable shock are associative in nature. Journal of Experimental Psychology: Animal Behavior Processes, 8 , 376-388.

Selugman, M. E. P. (1975). Helplessness: On depression, development, and death. San Francisco: W. H. Freeman.
Seligman, M. E. P., \& Maier, S. F. (1967). Failure to escape traumatic shock. Journal of Experimental Psychology, 74, 1-9.

Seligman, M. E. P., Rosellini, R. A., \& Kozak, M. J. (1975). Learned helplessness in the rat: Time course, immunization, and reversibility. Journal of Comparative \& Physiological Psychology, 88. 542-547.

SONODA, A. (1990). Effect of uncontrollable shock on subsequent appetitive discrimination learning in rats. Japanese Journal of Psychonomic Science, 8, 95-100. (Japanese, with English abstract)

TsUDA, A., IDA, Y., TANAKA, M. (1989). Differential effects of uncontrollability versus loss of stressor controllability on rat brain noradrenaline metabolism. Neurosciences, 15, 253-255. (Japanese, with English abstract)

Tsuda, A., Tanaka, M., Nishikawa, T., Hirai, H. (1983). Effects of coping behavior on gastric lesions in rats as a function of the complexity of coping tasks. Physiology \& Behavior, 30, 805-808.

Warren, D. A., Rosellini, R. A., Plonsky, M. \& DeCola, J. P. (1985). Learned helplessness and immunization: Sensitivity to response-reinforcer independence in immunized rats. Jourmal of $E x$ perimental Psychology: Animal Behavior Processes, 11, 576-590.

WEISS, J. M. (1971). Effects of punishing the coping response (conflict) on stress pathology in rats. Journal of Comparative \& Physiological Psychology, 77, 14-21.

Weiss, J. M., Goodman, P. A., Losito, B. G., Corrigan, S., Charry, J. M., \& BAILEY, W. H. (1981). Behavioral depression produced by an uncontrollable stressor: Relationship to norepinephrine, dopanine, and serotonin levels in various regions of rat brain. Brain Research Reviews, 3, 167-205

WIGHT, M. T., \& KATZEV, R. D. (1977). Noncontingent positive reinforcers retard later escape/avoidance learning in rats. Bulletin of the Psychonomic Society, 9, 319-321.

Williams, J. L., \& Maier, S. F. (1977). Transituational immunization and therapy of learned helplessness in the rat. Journal of Experimental Psychology: Animal Behavior Processes, 3, 240-252.

(Manuscript received May 16, 1990; revision accepted for publication February 24, 1991.) 